\title{
CONTRATACIÓN ELECTRÓNICA Y RELACIÓN DE CONSUMO. REGULACIÓN EN EL CÓDIGO CIVIL Y COMERCIAL DE LA REPÚBLICA ARGENTINA*
}

\author{
ELECTRONIC CONTRACTING AND \\ CONSUMPTION RELATION. \\ REGULATION IN THE CIVIL AND \\ COMMERCIAL CODE OF THE \\ ARGENTINE REPUBLIC
}

INGRID ROSAS VILLARRUBIA

Facultad de Derecho y Ciencias Sociales y Políticas

Universidad Nacional del Nordeste

ARGENTINA 


\title{
RESUMEN
}

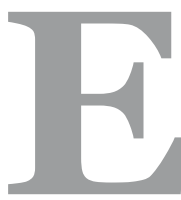

1 presente artículo aborda la contratación electrónica, dentro del régimen legal argentino con la reforma del código y su unificación en código civil y comercial. Se hará un análisis general del marco internacional, a su vez las conceptualizaciones para precisar el objeto de estudio y la referencia al mismo; para finalmente hacer un análisis de lo introducido por la reforma de nuestro código vigente, precisando algunos términos para un mejor desarrollo y comprensión del enfoque orientado de este articulo e introducir aspectos críticos en determinados momentos de su abordaje; que no pretende ser absoluto ni exhaustivo.

Palabras Clave: contratación electrónica, relación de consumo, código civil y comercial.

\begin{abstract}
This article deals with electronic contracting within the Argentine legal regime to reform the code and its unification in civil and commercial code, a general analysis of the international framework will , conceptualizations to clarify the object of study and reference thereto; to finally make an analysis of what introduced by the reform of our existing code, specifying certain terms for a better understanding of development and oriented in this article and introduce critical aspects at certain times in their approach. Treatment of electronic procurement addressed in this paper is not intended to be absolute or exhaustive.
\end{abstract}

Keywords: electronic contracting, consumer relationship, civil and commercial code.

\section{1.- ANTECEDENTES}

El proceso de globalización ha llevado al uso de diferentes herramientas para permitir la comunicación a través de la utilización de diversos canales, entre ellos el uso de canales informáticos, lo que permite la concertación contractual en modo espontáneo, superando las barreras de tiempo y distancia; la contratación a través de medios electrónicos ha sido una herramienta que ha facilitado la contratación internacional eliminando ampliamente las barreras de tiempo y localización.

\footnotetext{
*Este artículo fue producido en el marco del Proyecto de Investigación PI 16G 001 "La dimensión jurídica de la globalización. Impacto en el Nuevo Código" del Grupo de Investigación Deodoro Roca que integra la autora y dirige la Profesora (PH.D) Dra. Dora Esther Ayala Rojas. EI PI está acreditado por Resolución N 970/16 del C.S. de la UNNE.
} 
En la actualidad ha cobrado auge en el ámbito del comercio, el uso de transacciones en forma digital, la sociedad actual ha recibido el nombre sociedad de la información habido a que el proceso que transita es el uso de tecnología para diferentes actividades de los grupos humanos; entre ellas encontramos actividades de carácter contractual, de ahí la importancia del estudio de este fenómeno que tenemos presente actualmente y que presenta características particulares.

\section{METODOLOGÍA Y OBJETIVOS}

Se procederá a efectuar el análisis conceptual de la normativa existente según los procedimientos habituales en las ciencias jurídicas, identificando las diferentes posturas doctrinarias involucradas; por otra parte, se recurrirá al análisis comparativo con otros sistemas jurídicos con el propósito de identificar similitudes y diferencias conceptuales y doctrinarias, así como impactos en la aplicación concreta de la normativa y en la generación de casuística y su impacto en la protección del consumidor. Se trata de una investigación de carácter bibliográfico, cualitativo, descriptivo y de nivel exploratorio, con aplicación del método inductivo-deductivo.

\section{CONTRATACIÓN ELECTRÓNICA. NOCIÓN}

Al hablar de contratación electrónica estamos haciendo referencia a la modalidad de contratación por el uso de la tecnología en algunas de sus etapas; comprenda está la de perfeccionamiento o ejecución del contrato o en toda las etapas de contratación.

En cuanto a definiciones, se puede distinguir concepciones de carácter amplio que al hablar de contratación electrónica lo hacen con referencia a modalidades que implican el uso no solo de tecnologías por medio de ordenadores; si no comprende también las llevadas a cabo a través de telecomunicaciones, telefax o télex; tal es el caso de la ley modelo de la CNUDMI sobre comercio electrónico y su guía para incorporación en el derecho interno (UNCITRAL) en su art. 1, al circunscribir el ámbito de aplicación, se refiere a toda actividad comercial que se traduzca en el uso de mensaje de datos.

Por "mensaje de datos" se refiere a todo intercambio de información, comprensiva de intercambios electrónicos (EDI), correo electrónico, telefax, telegrama o telex.

En una concepción restrictiva se ciñe únicamente al EDI (intercambio electrónico de datos) y al correo electrónico es decir aquella modalidad que implique el uso de ordenador a ordenador para efectuar la contratación. Las reglas uniformes y código de conducta (URGETS) 
para el comercio electrónico define a comercio electrónico como "transacción comercial concluida mediante el intercambio de mensajes electrónicos...” circunscribiendo el objeto únicamente a las modalidades de comercio por el uso de ordenadores.

En una conceptualización de nuestro sistema legal, el contrato celebrado por medios electrónicos adopta una postura amplia y se lo trata como uno de los contratos celebrados a distancia, diferenciados de los contratos entre presente y ausentes. La modalidad de contratos a distancia se encuentra regulada dentro del ámbito de contratos derivados de la relación de consumo con referencia "modalidades especiales", enumerándose los medios por los cuales se celebra el contrato, no siendo taxativa dicha mención; por ende, existe un grado de flexibilidad por la incorporación de nuevas tecnologías que lleven a nuevas modalidades y que quedarán sometida a su régimen, habido a que adopta una postura abierta que permitirá responder a los cambios que se susciten a posteriori. Por ende, en nuestro sistema el ámbito de contratos a distancia, comprende aquellas modalidades de contratación que supone inmediatez en la respuesta pero caracterizado por la falta de presencia física simultanea de las partes o co-contratantes; entre ellos menciona los contratos electrónicos, los celebrados por medio de telecomunicaciones, postales, radio prensa o televisión. (Conf. art, 1105 del Código Civil y Comercial).

\section{DERECHO APLICABLE EN MATERIA DE CONTRATACIÓN ELECTRÓNICA. ÁMBITO INTERNACIONAL}

En el ámbito internacional no existen criterios unificados para aplicación de una normativa vigente; es así que tenemos una fuerte postura doctrinaria por el lado del derecho Europeo que enfatiza la necesidad de adopción unificada de una regulación legal en el ámbito internacional, para no obstaculizar los procesos de integración económica de un país a un mercado global, por establecimiento de pautas inflexibles que dificulten la materialización de esta forma de contratación.

La ley modelo de la UNCITRAL establece una regulación base para que se pueda contar con parámetros uniformes en la integración de una regulación específica para esta modalidad contractual; que cuenta con una guía para su incorporación por parte de los estados interesados. Dentro de la misma se recalca la importancia de la unificación de las normativas a los efectos de la integración de los países y que su existencia obedece no solo a la finalidad de establecer una ley marco que pretenda eliminar obstáculos jurídicos en materia de comercio electrónico, sino también de servir en materia de interpretación de otros instrumentos o convenios vigentes, como a la vez la actualización de regulaciones desactualizadas que presentan rigidez, debido a que no responden a las características mismas de la modalidad electrónica habido a que la legislación y archivo de información es anticuado o inadecuado. 
$\mathrm{Al}$ ser una regulación de carácter macro, establece que corresponde a los estados, con su incorporación, el establecimiento de reglamentos técnicos, cuando fuere necesario, dada la especialidad del tema, siempre respondiendo a la característica de flexibilidad; propone la aplicación del criterio del equivalente funcional; no deja sin efectos las disposiciones de los estados en materia de protección al consumidor teniendo primacía éstas por sobre las disposiciones de la ley modelo, salvo que el estado disponga lo contrario.

Europa cuenta con directivas, para diferentes cuestiones de carácter jurídico, las mismas son disposiciones normativas regentes para los estados que componen la Unión Europea. Dentro del ámbito de las directivas, a los efectos del presente análisis nos orientaremos al examen de la directiva 31/2000/PE, sobre comercio electrónico, que trata aspectos relacionados a los avances de la tecnología. Esta directiva brinda pautas normativas para evitar la existencia de obstáculos en el mercado interno que dificulten la libertad de circulación y libertad de establecimiento, propone a los estados la incorporación de legislaciones que permitan y no entorpezcan el proceso de contratación electrónica, a través de la creación de un marco general con cierto grado de flexibilidad; en cuanto a acuerdo de voluntades adopta la teoría de la recepción. Respecto a los consumidores, la misma impone la necesidad de que se establezcan legislaciones uniformes para dar seguridad en el marco del uso de este medio de contratación, sin dejar de lado la aplicación de disposiciones vigentes en materia de protección a los consumidores.

En el ámbito de integración regional (MERCOSUR), no existen disposiciones referentes a contratación electrónica; si encontramos diversas resoluciones vinculadas a los avances tecnológicos, tal el caso de la resolución vinculada al derecho de información en las transacciones ejecutadas a través de internet, la de certificación electrónica, la de reconocimiento de la eficacia del documento electrónico y la firma electrónica.

En Argentina tenemos, con la reforma del Código Civil y Comercial, su tratamiento en el título correspondiente a los contratos de consumo, como modalidades especiales artículos1104 y siguientes. Esto se ve complementado por la ley de firma digital, 25.506, que desarrolla el ámbito de su validez jurídica, definiendo las características que deben reunir los certificadores digitales. El ámbito del Código Civil y su reforma, con la incorporación de esta modalidad de contratación, será tratado más adelante.

\section{MODALIDADES DE CONTRATACIÓN ELECTRÓNICA}

Suelen distinguirse las modalidades online, que presuponen un proceso de contratación llevado a cabo en forma completa por vía digital, por ejemplo la adquisición de software; de la modalidad offline, que comprende que una de las etapas será realizada por el 
uso de un medio tradicional, v.gr transporte; ésta se da en los casos en que el contrato verse sobre bienes materiales.

Lorenzetti (2001, p. 173-174.) además realiza otra distinción en que se dan las modalidades de contratación; en una presupone la celebración de un contrato del consumidor con el proveedor de servicios y en una segunda instancia seria la contratación ya del consumidor con los proveedores de bienes y servicios por vía digital para la adquisición de los mismos a través de la red.

Por las partes que intervienen en la contratación electrónica se distinguen Business - Business (B2B) o Comercio de empresa a empresa (para el intercambio insumos o aprovisionamientos); Comercio Electrónico: Business - Consumer (B2C), entre proveedor y consumidor; Comercio Electrónico: Business - Government (B2G), entre empresarios y el Gobierno y Comercio Electrónico: Consumer-Consumer ( $\left.\mathrm{C}_{2} \mathrm{C}\right)$, entre consumidores.

$\mathrm{Al}$ presente trabajo interesa el $\mathrm{B} 2 \mathrm{C}$ la relación entre los proveedores y consumidores, materia que regula nuestro Código unificado, en consonancia con el microsistema de Defensa del Consumidor, constituido por la Ley 24.240, la Ley 25156, de Defensa de la Competencia, y las leyes especiales por actividad, tales como las de tarjetas de crédito, seguros, entre otras. Este espectro surge de la teoría del diálogo de fuentes, adoptada por el Código Civil y Comercial.

Para hacer una aclaración, en el ámbito de las modalidades con intervención de las diferentes partes, cada uno tiene en materia de regulación diferentes legislaciones con diferentes principios v. gr el de contratación entre empresas B2B que se sigue en líneas generales por las URGETS (reglas uniformes y código de conducta para el comercio).

Las mentadas ut supra serían las principales modalidades de contratación, que encontraremos presente en el ámbito del comercio electrónico internacional.

\section{LA CONTRATACIÓN ELECTRÓNICA EN EL CÓDIGO CIVIL Y COMERCIAL ARGENTINO}

\subsection{Noción de contratación electrónica en Argentina}

En nuestro ámbito nacional podemos mentar definiciones, v.gr Lorenzetti (2001, p. 173-174) quien brinda la siguiente noción: "El contrato electrónico se caracteriza por el medio empleado para celebrarlo, cumplirlo o ejecutarlo, sea en una o en las tres etapas en forma total o parcial... El contrato puede ser celebrado digitalmente en forma total o parcial: en el primer caso, las partes elaboran y envían sus declaraciones de voluntad (intercambio electrónico de datos o por una comunicación digital interactiva); en el segundo, solo uno de estos aspectos es digital: una parte puede elaborar su declaración y luego utilizar el medio digital para enviarla; se puede enviar un mail y recibir un documento escrito para firmar. Puede ser cumplido total o parcialmente en medios digitales: en el primer caso, se transfiere un bien digitalizado y se paga con "moneda digital”; en el 
segundo, se envía un bien digital y se paga con un cheque bancario, o se envía un bien físico por un medio de transporte y se paga con transferencias electrónicas de dinero".

Feldstein de Cardenas (2008, p.139) los define como "aquellos acuerdos de voluntades celebrados mediante la utilización de medios electrónicos, telemáticos o informáticos, que, sea en su conformación, desarrollo o extinción, poseen elementos extranjeros objetivamente relevantes desde la mira de un ordenamiento jurídico determinado”.

En nuestro Código, como habíamos mencionado en los primeros títulos del presente trabajo, se encuentran regulados dentro del tratamiento de los contratos a distancia, con referencia a la contratación emergente de contratos de consumo, dentro del marco de la relación de consumo. Incorpora una concepción amplia, debido a que se está frente a conceptos abiertos permitiendo, en el caso de aparición de nuevas modalidades de contratación electrónica, que la mismas puedan ser abarcadas por la presente regulación, respondiendo también a lo que se plantea a nivel internacional en cuanto que no solo abarcara el EDI (intercambios electrónicos), si no refiere también a una gama de aplicaciones que se utilice en el ámbito del comercio, por ende el Código, al tratar como contratos a distancia con una enumeración no taxativa, genera una categoría amplificada sin especificar a qué tipos de contratos se aplica (Rivera 2014, p.755.).

\subsection{Relación de consumo. Sujetos}

Al hablar de relación de consumo la misma hace referencia al vínculo jurídico que emerge entre el proveedor y consumidor (Art. 1092 CCC). La idea de la relación de consumo dentro de nuestra legislación se fue gestando en una etapa primero de objeto restringido siendo que la misma en los orígenes de la ley 24.240 se aplicaba a la relación emergente de la contratación onerosa, con la reforma constitucional y posterior incorporación del artículo 42, la relación de consumo pasa a ser un concepto constitucional, se da la modificación de la ley 24.240, en 2008, la cual obedece a la mencionada reforma, y se amplía la noción habido que comprende la protección a todos los vínculos jurídicos entre el proveedor con el consumidor o usuario (art 3 LDC). Con la sanción del Código Civil y Comercial se va a redoblar en algún aspecto la protección al consumidor, siendo que el mismo no solo encuentra amparo en la vigente ley de defensa del consumidor (ley 24.240), sumado a esta cuenta con la proteccion en el marco del Código en referencia a modalidades de contratación no contempladas en forma exhaustiva por nuestra legislación del consumidor (LCD).

El presente trabajo se centra en una de las nuevas modalidades reguladas, la contratación por vía electrónica. Como nota a tener en cuenta, las normas del consumidor no se agotan con la existencia de dicha normativa, las mismas pueden ser reforzadas, teniendo en cuenta que el régimen protectorio regulado en el ámbito constitucional tiene la característica de integral por ende del ejercicio del poder de policía por parte de las provincias se debe procurar esta protección integral siendo que se encuentran habilitados por el ejercicio de esta facultad de policia, 
a dictar normas complementarias como lo mismo pueden hacer las autoridades municipales (Fariña 2009, p.55-69).

La regulación surge como respuesta a la necesidad de protección de los derechos en el ámbito del consumo habido a que estos contratos generalmente se presentan en forma masiva, conocida como contratos en masa y con cláusulas predispuestas; por ende, esta regulación lo que hace es marcar un límite al proveedor frente a las cláusulas pactadas por éste aprovechando de su posición fuerte en el mercado, por eso es que la regulación pasa a ser de interés general, siendo que el Estado actúa como protector por medio de la legislación otorgando al consumidor una herramienta para recurrir en caso de que sus intereses se vean afectados.

A la vez, brinda un límite del cual no puede excederse el proveedor para ejercer su derecho en forma abusiva, brindando protección a la parte más débil dentro del vínculo que se suscita; en este caso es el consumidor o usuario.

Hay que también tener presente a los efectos, la diferenciación que tiene nuestra legislación en el consumo habido a que por un lado tenemos los derechos del consumidor que son de incidencia colectiva y los que darán lugar a un reclamo subjetivo particular; para hacer referencia en forma sintética, al hablar de derechos de incidencia colectiva son aquellos que dan lugar a un reclamo generalizado por afectación del interés de un grupo considerable y determinado. No toda violación implica una lesión a los derechos de incidencia colectiva, esta distinción no se basa en el derecho conculcado o violado únicamente el cual debe ser de dimensión social e interés público, sino también al grado de tutela jurisdiccional que se busca (Rivera 2008, p.733-737.); las dos vías en nuestra legislación se encuentran habilitadas la del reclamo individual y la del reclamo colectivo.

Nuestra legislación define al consumidor como la persona física o jurídica que adquiere bienes y servicios como destinatario final. El término destinatario final presupone que la adquisición del producto o servicio está afectado al consumo sea del adquirente o de su grupo familiar y social, sin que pueda ser reinsertado en la cadena de producción-comercialización (Art. 1 LDC; Art1092 CCy Com). Por su parte, Proveedor es la persona física o jurídica de carácter público o privado que desarrolla de manera profesional u ocasional actividades vinculadas al ámbito del comercio (producción, distribución, importación, etc.).

Respecto al contrato de consumo, en nuestro régimen se fortalece la regulación con la vigencia del Código Civil y Comercial, se encuentra definido por dicho cuerpo legal como el celebrado por un proveedor y un consumidor, que tiene por objeto la adquisición, uso o goce de bienes o servicios por el consumidor o usuario para su uso privado o de su grupo familiar o social (Art. 1093 CC y Com). Lorenzetti, en el Anteproyecto de Código Civil y Comercial ha expuesto que en nuestra regulación actual se diferencian dos marcos contractuales, a los efectos de dirimir los conflictos y confusiones y el nivel de protección brindado, esta fragmentación en palabras de Lorenzetti “... se construye sobre la necesidad de neutralizar la vulnerabilidad producida por la asimetría de información, inferior capacidad técnica, y 
dispar capacidad jurídica y de negociación que suele presentarse entre los contratantes en los contratos de consumo".

\subsection{Contratación electrónica y relación de consumo. Prueba, forma. Oferta y revocación de la aceptación}

En el régimen de la contratación electrónica, dada la modalidad de la misma, se encuentra la característica de no constar con un instrumento por escrito; por ende, si bien nuestra legislación requiere la presentación del contrato escrito a los efectos de la prueba, con la reforma se incorpora el artículo 1106, que otorga un margen flexible. En el ámbito del uso de la tecnología, se considera satisfecho el requisito si consta en un soporte electrónico o tecnología similar, es lo que se conoce como mensajes de datos de acuerdo a la ley modelo de la UNCITRAL “...por mensaje de datos se entenderá la información generada, enviada, recibida o archivada o comunicada por medios electrónicos, ópticos o similares...”. Es decir que, dada la modalidad de esta clase de contratación, se inclina el Código por un criterio amplio, permitiendo una actualización de su normativa por las circunstancias tecnológicas actuales. No obstante, ésta es una posibilidad que le brinda la ley al consumidor, pero puede exigir al proveedor que el contrato conste por escrito para su mayor seguridad; en cuyo caso, el proveedor se encuentra obligado a suministrar dicho documento. Con la reforma del Art 4 de la ley de defensa del consumidor en Junio del 2016, a través de la ley 27.250 en referencia al cumplimiento de la obligación de información que debe ser suministrada por el proveedor al consumidor, la misma debe ser precisada en forma cierta, clara y detallada en referencia a las características esenciales. Esta reforma establece la obligatoriedad de suministra información al consumidor en soporte físico, salvo manifestación expresa del consumidor o usuario de usar otro medio alternativo de comunicación puesta a disposición por el proveedor.

Rivera (2014, p.756-757) nos habla de la finalidad tuitiva que tiene el contrato escrito respecto del consumidor y que si el consumidor prefiere que dicha relación se plasme en un documento por escrito la misma debe ser obligatoria, teniendo en cuenta que el ámbito de la celebración del contrato electrónico por el uso de la tecnología para algunos consumidores le es ajena o complicada. Esto se ve solucionado con la posterior reforma de la ley de defensa en el Art. 4 mentado ut supra.

La importancia del contrato escrito y la firma en soporte físico, en palabras de Rivera es que el mismo, otorga mayor seguridad para efectuar el reclamo en caso de incumplimiento; la firma electrónica también satisface este requisito, en remisión al art 288 CC y Com., siempre que asegure autoría e integridad. Los parámetros están fijados en nuestra ley de firma digital, $\mathrm{N}^{\mathrm{a}} \mathbf{2 5 . 5 0 6}$, en la que hay firmas que gozan de autenticidad expedida por certificadores digitales, los cuales tienen fuerza probatoria. Basta que el consumidor, al contratar, constate dentro del ámbito de acceso a la página que ésta cuente con la respectiva certificación a los efectos de garantizarse el cumplimiento. 
Es lógico que Internet, al ser una red abierta, no puede ser agotada en cuanto a control de manera exhaustiva dada la facilidad de acceso y la internacionalidad, por ende por eso existen instrumentos de los cuales el consumidor puede valerse para contratar dentro de un margen de garantía, v. gr sería el caso de contrataciones con paginas certificadas por certificadores licenciados, lo cual le otorga mayor seguridad jurídica y en caso de incumplimiento el reclamo puede ser cursado en forma y con resultado efectivo.

El proveedor está obligado a informar el contenido mínimo del contrato, informando riesgos, posibilidad de revocar y brindar datos para la correcta utilización del medio tecnológico en uso. (Art. 1107 CCC); en cuanto a los riesgos se debe dejar claro quién debe soportar los mismos.

En referencia al contenido mínimo exigible del contrato podemos remitirnos a la LDC en su art 10:

“ARTICULO 10. - Contenido del Documento de Venta. En el documento que se extienda por la venta de cosas muebles, sin perjuicio de la información exigida por otras leyes o normas, deberá constar:

a) La descripción y especificación de la cosa;

b) El nombre y domicilio del vendedor;

c) El nombre y domicilio del fabricante, distribuidor o del importador cuando correspondiere; (Inciso observado por el Art. $1^{o}$ del Decreto Nacional $N^{o}$ 2089/93 B.O 15/10/1993)

d) La mención de las características de la garantía conforme a lo establecido en esta ley;

e) Los plazos y condiciones de entrega;

f) El precio y las condiciones de pago.

La redacción debe ser hecha en idioma nacional, ser completa, clara y fácilmente legible, sin reenvíos a textos o documentos que no se entreguen previa o simultáneamente. Un ejemplar debe ser entregado al consumidor. Cuando se incluyan cláusulas adicionales a las aquí indicadas o exigibles en virtud de lo previsto en esta ley, aquéllas deberán ser escritas en letra destacada y suscritas por ambas partes...”.

En materia de riesgos, tal surge de la interpretación que se hace al texto, abre la posibilidad de traslado de los riesgos hacia el consumidor; no obstante, entraría en contradicción con el sistema protectorio de las políticas de defensa del consumidor adoptadas hasta ahora en nuestra legislación.

La cláusula de traslación de los riesgos puede resultar abusiva por implicar una limitación a la responsabilidad del proveedor, la misma seria ineficaz en virtud de lo dispuesto en el art 37 LDC y 1117 CC y Com. (Rivera 2014, p.757-758). 
La oferta por medios electrónicos va a mantener su vigencia por el plazo de duración fijado por el proveedor. Se da aplicación a lo señalado previamente por la LDC que las ofertas en cuanto a que la duración debe tener un plazo determinado en forma clara, debiendo contener en forma expresa modalidades, condiciones o limitaciones; además, incorpora la situación que, de no estar estipulado el plazo será por el tiempo que perma-nezcan accesibles al destinatario.

A su vez, establece la obligación al proveedor de confirmar la llegada de la aceptación, si bien nuestro Código en materia de perfeccionamiento de los contratos con modalidades entre ausentes adopta la teoría de la recepción, haciendo extensiva su adopción para las modalidades por vía electrónica. Incorpora la obligación al proveedor de confirmar la recepción de la aceptación, a los efectos de evitar conflictos por la no recepción de la aceptación canalizada por medios electrónicos; por tanto, la característica de la presente modalidad responde a la obligación impuesta.

En lo referido al lugar de cumplimento (art 1109 CC y Com.), en principio, en materia de contratación rige la autonomía de voluntad de las partes pudiendo pactar éstas libremente el lugar de cumplimiento y la jurisdicción y legislación aplicable; no obstante, en materia de contratación electrónica el ámbito de la jurisdicción se encuentra circunscripto al lugar de cumplimiento, donde recibió o debió recibir la cosa, quedando vedada la prórroga de jurisdicción.

El presente artículo solo comprende una modalidad de contratación electrónica, aquellas que suponen la entrega física del bien, dejando de lado las modalidades de contratación en donde se efectúa todo por vía digital. Una de las dificultades de esta modalidad de contratación y que debe ser resuelta es la materialización del lugar, la misma encuentra dificultad dada las características de incorporalidad; se presenta el obstáculo de conocer y determinar los recorridos que siguen las informaciones digitales, la localización de quienes operan en internet es desconocida; la localización del sitio en caso de ser posible en muchos casos aparece encriptada o puede darse la existencia de mirrors; la navegación se hace de manera anónima y el número de destinatarios es de carácter infinito por los bajos costos de accesibilidad del internet (Scotti 2012, p.130-135).

Las dificultades deben ser tratadas; siendo que queda sin margen regulatorio al cual recurrir en caso de conflicto en materia de contratación electrónica efectuada en su totalidad por vía digital. Es importante determinar la ley aplicable y el ámbito jurisdiccional al que se somete para dar respuesta en caso de controversia de partes.

En cuanto a la posibilidad de revocación consagrada por el artículo 1.110 y ss., se encuentra también contemplado en el art. 34 LDC, como una facultad con la que cuenta el consumidor que contrate bajo la modalidad especial de contratos celebrados fuera del establecimiento mercantil, de dejar sin efecto el contrato y sin necesidad de justificar el retiro de dicha aceptación. El mismo tiene establecido un plazo de 10 días a partir de la celebración del contrato como principio general, no obstante en caso de que la entrega es posterior a la aceptación el plazo se computa a partir de la entrega, compartiendo la crítica de Rivera (2014, p.762-764). Dicho autor pone de manifiesto la disonancia contra el régimen protectorio, tal es así que la aceptación posterior a la entrega una vez vencido el plazo de los 10 días 
daría lugar a la eliminación de la posibilidad de revocar esa aceptación, por eso se deberá computar desde el perfeccionamiento, con remisión a lo dispuesto en el art 34 LDC, donde el plazo comienza a correr desde la aceptación o la entrega, de acuerdo a lo que suceda en último término.

El ejercicio del derecho de revocación es discrecional. La naturaleza del ejercicio del derecho de revocación es asimilable al de un contrato bajo condición suspensiva hasta tanto no transcurra el plazo de diez días del vencimiento de la revocación (Rivera 2014, p. 174-176), dándose en dicho periodo el traslado de los riesgos al proveedor. Los efectos se producen ex tunc de la misma forma que en el caso de la compraventa a satisfacción del comprador (art. 1160 CC y Com.), respecto a la devolución, su imposibilidad no priva al consumidor del derecho de revocar. En caso de mediar culpa del consumidor, deberá pagar al proveedor el valor del bien al momento de revocar.

El ejercicio del derecho de revocar es gratuito, además que impone la obligación que se restituyan al consumidor los gastos útiles y necesarios que realizó en la cosa, recae sobre el consumidor la única obligación de poner el bien a disposición del vendedor, en modo alguno debe cargar con los gastos de trámite y devolución (Calderón 2014, p. 74).

\section{DISCUSIÓN Y RESULTADOS}

Se hace necesaria una regulación que responda a los parámetros actuales frente a esta modalidad de contratación, la cual presenta características particulares, distintas a la restantes. Si se da lugar a una aplicación analógica, siguiendo a la pauta tradicional, la misma resultará negativa en cuanto a inserción en el mercado económico del país. Las barreras existentes siguiendo un ámbito tradicional, subyacen en la falta de flexibilidad de sus pautas, las cuales resultan contrarias a esta nueva modalidad de contratos. La postura que mejor se adecua frente a esta modalidad de contratación que responde a la globalidad del mercado actual, es la que enmarca la necesidad de la creación de una ley macro específica, que contenga principios y parámetros vinculados a esta modalidad, vgr., el del domicilio virtual para las notificaciones procesales a los efectos que ellas tengan eficacia jurídica y puedan cursarse a través de medios informáticos. Las dificultades actuales, a superar, tienen vinculación con la seguridad en las modalidades de pago; la incertidumbre en materia de legislación aplicable en cuestiones de contratación electrónica internacional; la falta de información de procedimientos adecuados y seguros para concertar negocios por medios informáticos sin terminar expuestos a fraudes o estafas cibernéticas; en cuanto entrega, pago, garantía y servicio post-venta se hace necesario fijar límites y pautas predominantes para otorgar margen de seguridad al consumidor. 


\section{CONCLUSIÓN}

El Código Civil y Comercial obvió referirse específicamente a los contratos electrónicos debiendo solucionar las cuestiones que los atañe por vía de la analogía. Sin embargo, podría inferirse que debería legislarse mediante un microsistema especial en torno a esta cuestión, dada la entidad y proliferación de su uso en el comercio actual. La ley especial que se dicte deberá atender a los principios específicos y, además, prever en forma diversa los contratos entre empresas y los de consumo; sin desconocer que, probablemente, todos se formalicen mediante adhesión a condiciones generales.

\section{REFERENCIAS BIBLIOGRÁFICAS}

Calderón, Maximiliano R.- Márquez, José F., "El arrepentimiento en el derecho del consumo”, La ley, 2009-C, 742. Código Civil y Comercial Argentino.

De Lorenzo, Federico M., "Derecho al arrepentimiento en la contratación comercial”, en Picasso -Vazquez Ferreyra, cit., 2009.

Directiva 2000/31/ce del Parlamento Europeo y del consejo relativa a determinados aspectos jurídicos de los servicios de la sociedad de la información, en particular el comercio electrónico en el mercado interior (directiva sobre el comercio electrónico) 8 de junio de 2000.

Farina, Juan M.. "Defensa del consumidor y del usuario" 4ta Edición. Bs As. Editorial Astrea. 2008, pg.- 1-46.

Fildstein de Cardenas, Sara; Scotti, Lucia B.; Rodríguez, Mónica S.; Medina, Flavia A. “Contratación electrónica internacional”. Argentina. Año 2008.

Ley Modelo de la CNUDMI sobre comercio electrónico con su guía para la incorporación al derecho interno 1996.

Lorenzetti, Ricardo, Comercio electrónico, Buenos Aires, Abeledo Perrot, 2001.

Medina, Graciela; Rivera, Julio C. "Código civil y comercial argentino comentado" Tomo III. Argentina. Año 2014. 
Scotti, Luciana B. "La contratación electrónica en el código civil argentino: una mirada al derecho internacional”. Lecciones y ensayos $N^{\circ}$ 90. Pág. 129-169. Argentina.

\section{CURRICULUM VITAE}

\section{Ingrid Rosas Villarrubia}

Abogada, Facultad de Derecho y Ciencias Políticas y Sociales, Universidad Nacional del Nordeste (UNNE), Argentina, 2016. Adscripta cátedra del derecho comercial I, Facultad de Derecho y Ciencias Políticas y Sociales, UNNE, Argentina.

rosasvillarrubiaing@gmail.com 